\title{
ANÁLISE DA VIABILIDADE DE IMPLEMENTAÇÃO DO ENSINO DA METODOLOGIA BIM NO CURSO DE ENGENHARIA CIVIL: CASO DA UNIVERSIDADE FEDERAL DO CARIRI
}

Thayná Ribeiro da Silva - silvathayna20@outlook.com

Universidade Federal do Cariri

Juazeiro do Norte - Ce

Fábio Cristian Alves Pinheiro Filho-fabio.cristian@aluno.ufca.edu.br

Universidade Federal do Cariri

Juazeiro do Norte - Ce

Gabriel Vieira Ribeiro-gabriel.icasa@gmail.com

Universidade Federal do Cariri

Juazeiro do Norte - Ce

Ana Verônica Gonçalves Borges - ana.borges@ufca.edu.br

Universidade Federal do Cariri

Juazeiro do Norte - Ce

\section{Resumo}

A modelagem da informação da construção (Building Information Modeling - BIM) tem um potencial inovador e está ganhando cada vez mais espaço dentro da construção civil por oferecer um conjunto de tecnologia, exatidão e facilidade, agregando ao manejo da obra uma alta produtividade e eficiência. Porém, muitas universidades têm como obstáculo, implantar o ensino do BIM e formar profissionais capazes de suprir as demandas do mercado de trabalho. Em virtude disso, e visando superar tal discrepância, este artigo denota uma análise detalhada de como poderia ocorrer a implementação do ensino do BIM, em particular, no curso de Engenharia Civil da Universidade Federal do Cariri (UFCA). Inicialmente, é apresentado um estudo das dificuldades de inserir tal ensino na graduação de profissionais desta área. Logo em seguida, é feita uma análise tanto das disciplinas ofertadas no curso, como das dificuldades apresentadas pelos docentes para ocorrer esta inserção. E assim, estabelecendo parâmetros significativos, juntamente com uma metodologia para a integração do ensino do BIM na grade curricular em atuação. Ficando notório observar que logo nos primeiros semestres é possível implantar a metodologia em disciplinas básicas.

Palavras-chave: BIM. Ensino. Graduação.

\section{ANALYSIS OF THE FEASIBILITY OF IMPLEMENTING THE TEACHING OF THE BIM METHODOLOGY IN THE CIVIL ENGINEERING COURSE: CASE OF THE FEDERAL UNIVERSITY OF CARIRI}

Abstract: 
Building information modeling (BIM) has an innovative potential and is gaining more and more space within the civil construction sector by offering a set of technology, accuracy, and ease, adding to the management of the work high productivity and efficiency. However, many universities have the obstacle of implementing BIM education and training professionals capable of meeting the demands of the labor market. As a result, and to overcome such discrepancy, this article denotes a detailed analysis of how the implementation of BIM teaching could occur, in particular, in the Civil Engineering course at the Federal University of Cariri (UFCA). Initially, a study of the difficulties of inserting such teaching in the graduation of professionals in this area is presented. Soon after, an analysis is made of both the subjects offered in the course, as well as the difficulties presented by the teachers to occur this insertion. And so, establishing significant parameters, together with a methodology for integrating BIM teaching into the current curriculum. It is notorious to note that in the first semesters it is possible to implement the methodology in basic subjects.

Keywords: BIM. Teaching. Graduation.

\section{INTRODUÇÃO}

Barison (2015) indica que "os estudantes ainda não estão sendo preparados para ingressar no mercado de trabalho com conhecimentos e habilidades em BIM". O mesmo indica em sua argumentação que a falta de qualificação do corpo docente sobre a temática BIM e a ausência nas grades curriculares, surgem como uma das principais causas dessa uniformidade no perfil dos profissionais a serem formados pela instituição. Diante dessa problemática foi feito um estudo em diferentes níveis com o intuito de sanar a carência do BIM no curso de Engenharia civil da UFCA, visto que o ano de 2021 representa um marco na implantação do BIM no Brasil, pois o Decreto ${ }^{\circ} 10.306$, de 2 de Abril de 2020 estabelece a obrigatoriedade do uso do BIM nas obras públicas de grande relevância para disseminação da referida metodologia.

A Universidade Federal do Cariri é uma universidade brasileira, localizada no sul do Estado do Ceará, que possui cinco campi espalhados pela região do Cariri e desde 2006 já apresentava o curso de Engenharia Civil na região.

\section{OBJETIVOS}

Este artigo possui como objetivo geral identificar questões relacionadas à implementação do BIM nas universidades e sua influência na formação profissional dos estudantes da área da construção civil. Existe uma demanda crescente por parte das empresas na utilização do paradigma e junto a isso há a necessidade da adaptação dos profissionais na área. Como primeiro passo para tal feito, a adoção de uma adequação no modelo curricular de ensino seria um ponto de partida.

O presente estudo visa antecipar a inclusão do BIM na universidade através de algumas disciplinas que possuem conteúdos consoantes com a metodologia e, consequentemente, obter uma melhor qualificação dos profissionais da construção civil na região do Cariri.

\section{ENSINO-APRENDIZAGEM DE BIM E SUA ADOÇÃO NAS UNIVERSIDADES}


Em busca de renovação constante na área da construção civil, surge então como principal alternativa o BIM e suas demais vertentes. Em uma das definições mais usuais, trata-se de um processo que permite a gestão e integração de informações nas diferentes etapas construtivas de uma edificação. Segundo Eastman, Teicholz, Sacks e Liston (2014) o BIM é uma série de processos, métodos, softwares e tecnologias usadas para facilitar e maximizar a comunicação entre os diferentes profissionais que agregam quaisquer edificações e seus diferentes níveis de execução e/ou planejamento.

Com o crescente interesse da indústria de Arquitetura, Engenharia e Construção (AEC) pela utilização do BIM, a procura por profissionais com conhecimento e domínio sobre o paradigma também se torna maior. No entanto, a falta de pessoal capacitado ou que conheça os benefícios da metodologia, acaba por criar dificuldades na aplicação da mesma. É portanto consensual que cada vez mais profissionais na área da construção necessitam adquirir conhecimentos e competências para colaborar e se comunicar através das ferramentas BIM, o que necessariamente irá englobar o processo educativo e a formação dos engenheiros civis (LINO; AZENHA; LOURENÇO, 2012)

Apesar dos desafios mencionados, algumas universidades obtiveram sucesso na implantação do BIM na grade curricular. Segundo Barison e Santos (2011), nos Estados Unidos, a Georgia Institute of Technology foi precursora do movimento da introdução das ferramentas BIM ao realizar pesquisas desde a década de 1990, tendo sido seguida pela maioria das demais universidades americanas a partir de 2003.

No âmbito nacional, de acordo com Ruschel, Andrade e Morais (2013), a difusão das discussões sobre BIM foi impulsionada pela crescente preocupação com a implementação da Modelagem de Informação da Construção nos cursos de Arquitetura e Engenharia Civil. Algumas universidades brasileiras, citadas pelos autores, que já estão trabalhando conteúdos referentes ao BIM em seus currículos com sucesso, são a Universidade Federal de Alagoas (AL), o Centro Universitário Barão de Mauá (SP), a Universidade Presbiteriana Mackenzie (SP), a Universidade Federal de São Carlos (SP) e a Universidade Estadual de Campinas (SP).

\section{METODOLOGIA}

O presente trabalho constitui uma pesquisa científica do estudo de caso sobre ensino do BIM na UFCA, na qual "é encarado como o delineamento mais adequado para a investigação de um fenômeno contemporâneo dentro de seu contexto real, onde os limites entre o fenômeno e o contexto não são claramente percebidos" (apud GIL, 2010, p.37). A metodologia adotada para consecução desta pesquisa contemplou as seguintes etapas:

a) Etapa I - descrição do caso estudado: análise das disciplinas de modo a identificar quais possuíam a viabilidade de implantar o ensino do BIM. Além disso, a realização de uma breve entrevista com os docentes buscando identificar os possíveis obstáculos, que os mesmos constataram para inserir o ensino do BIM nas disciplinas que ministram.

b) Etapa II - análise dos resultados: utilizando dentro das disciplinas do curso, um método de implantação do BIM, torna-se possível verificar a solução mais adequada para viabilizar a inserção da metodologia na Universidade, considerando suas particularidades. 
O estudo das disciplinas foi realizado por meio de uma adaptação dos métodos estabelecidos por Checcucci (2014), onde as análises das ementas servem de entrada para o preenchimento de campos com cores identificando, em cada disciplina, como cada item foi avaliado de acordo com seu potencial de inserção de BIM (Figura 1):

Figura 1 - Sistema de representação utilizado para registrar as análises das ementas das disciplinas

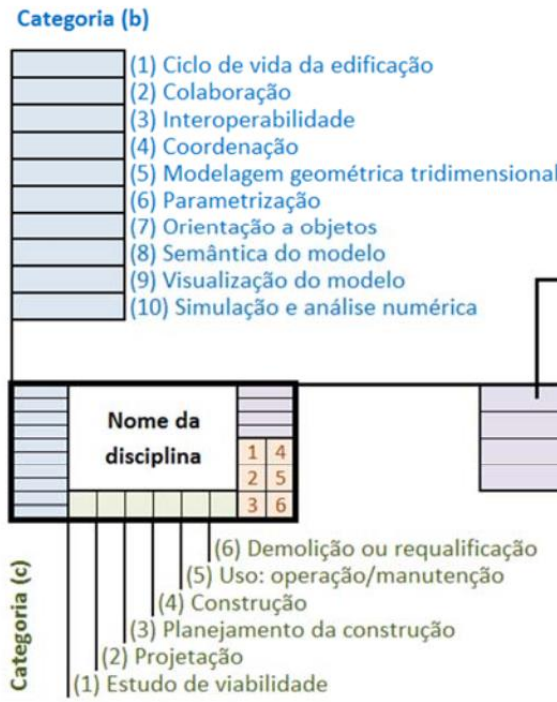

\section{Categoria (a)}

(1) Não se visualiza nenhuma interface com o paradigma (2) Depende do foco dado pelo professor (3) Tem interface com o paradigma

(a) Relação componente curricular x BIM (b) Pode trabalhar algum conceito importante do BIM Pode trabalhar com alguma etapa do ciclo de vida da edificação d) Pode trabalhar alguma disciplina de projeto

Fonte - Adaptado de Checucci (2014)

\section{RESULTADOS E DISCUSSÕES}

Através das pesquisas bibliográficas e análises das ementas de todas as disciplinas obrigatórias do curso, obteve-se o resultado explicitado na figura 2, onde foi mapeado todas as possibilidades de ensino do BIM, analisando cada disciplina de forma particular, conforme o método de Checcucci (2014). Na figura 3 tem-se apenas as disciplinas que possuem interface explícita com o paradigma.

Figura 2 - Componentes que possuem interface com o paradigma BIM

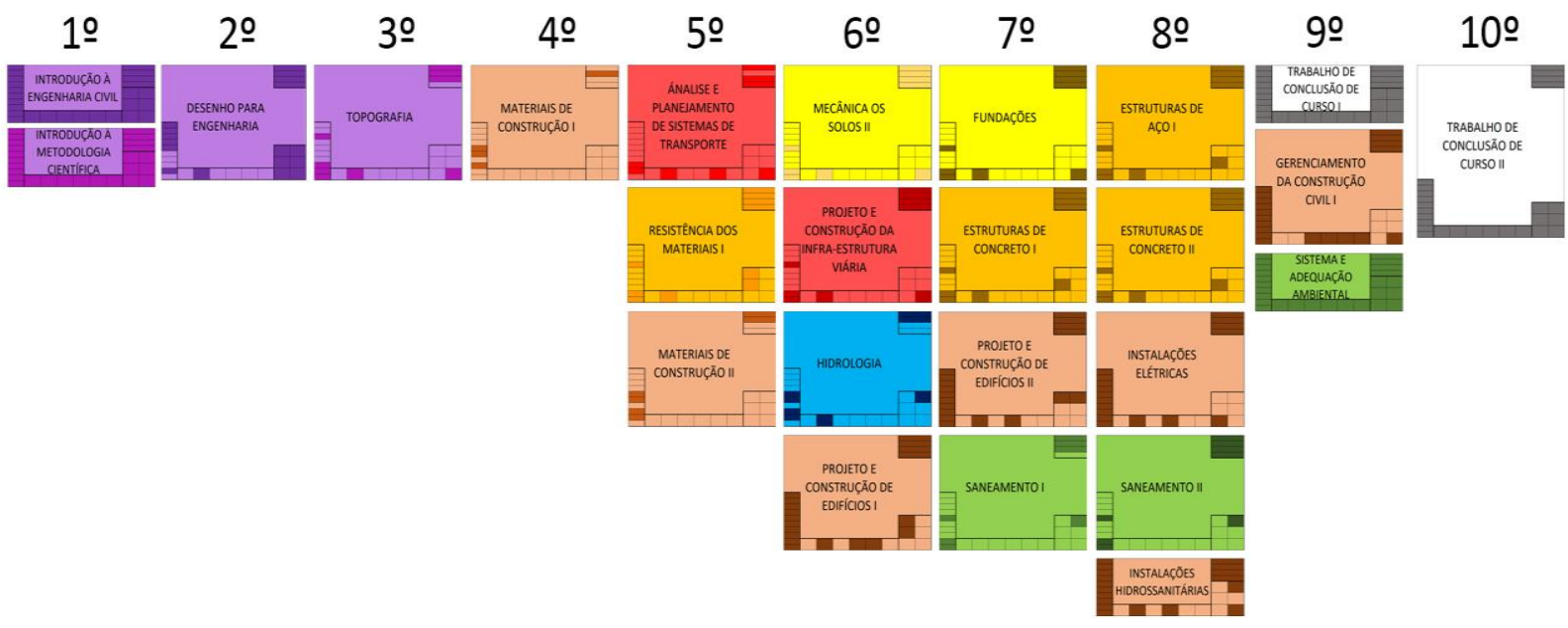

Fonte - Os autores 
Figura 3 - Componentes que possuem interface explícita com o paradigma BIM

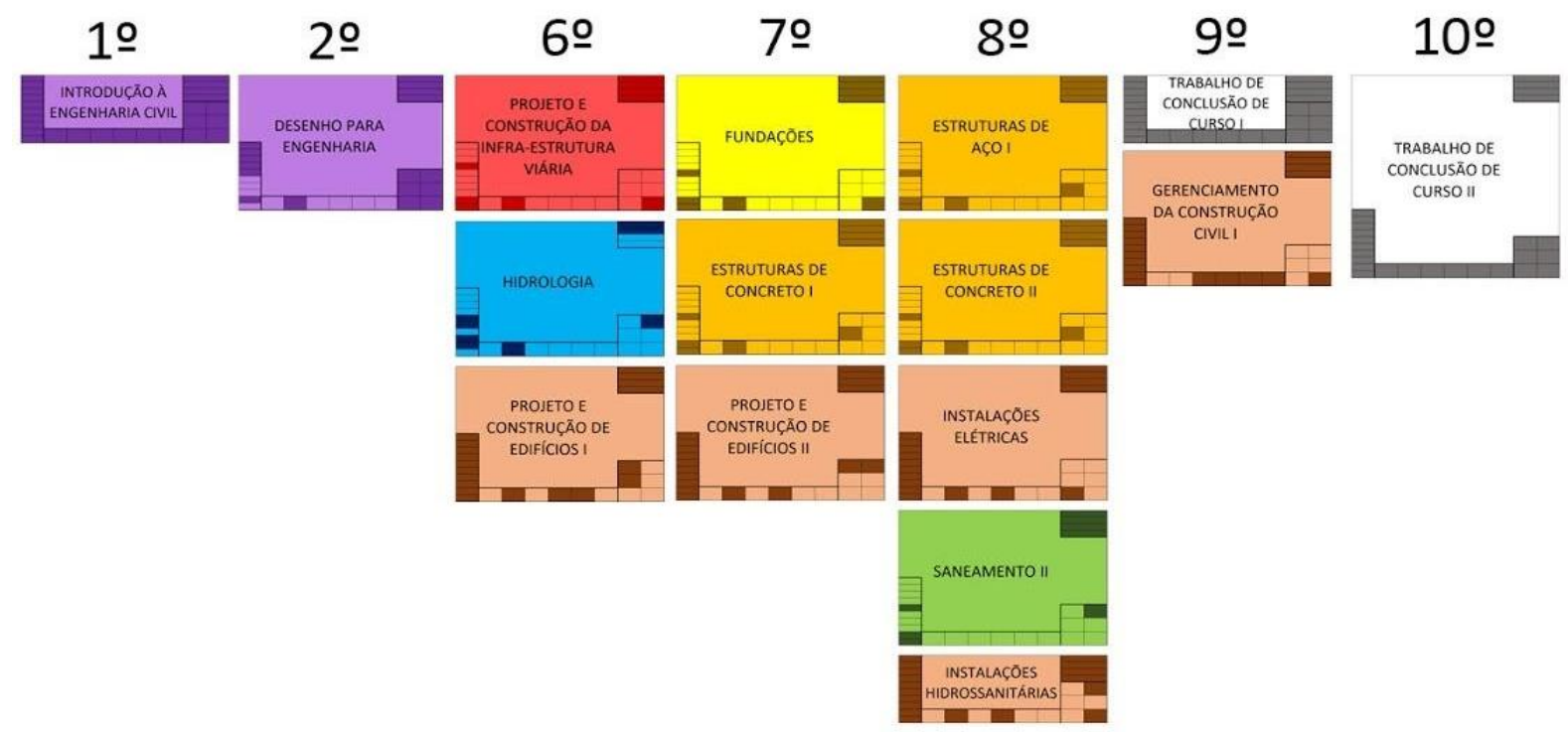

Fonte - Os autores

Verifica-se que mesmo no Núcleo Básico, os alunos podem ter contato com a metodologia BIM através de 4 disciplinas. Dentre estas, Introdução a Engenharia Civil pode abranger todos os aspectos do BIM e suas aplicações ao apresentar para os alunos os fundamentos de projetos e as áreas de atuação do Engenheiro Civil. Já a disciplina de Introdução à Metodologia Científica pode utilizar artigos sobre o BIM para ambientar os novos estudantes ao curso, mesmo este não sendo o seu foco. Além disso, na ementa de Desenho para a Engenharia é possível implementar os conceitos de modelagem básica da metodologia BIM e ferramentas podem ser apresentadas na disciplina de Topografia, como forma de agilizar a coleta de dados em campo e o processamento dessas informações.

$\mathrm{Na}$ área de Recursos Hídricos em apenas 1 disciplina foi encontrada relação com o paradigma BIM, porém sua aplicação é essencial, visto que a disciplina de Hidrologia lida com projetos de grande porte, nas quais através de softwares BIM os projetos são realizados de forma mais precisa e com parâmetros mais próximos à realidade.

A metodologia BIM pode ser empregada na área de Saneamento a partir do $7^{\circ}$ semestre e conta com 3 disciplinas possíveis. Em Saneamento I e II, caso haja projetos a serem realizados, é possível mostrar como a modelagem desse projeto pode ser realizada em diferentes softwares, caso contrário o professor pode exemplificar tal metodologia. Já em Sistema e Adequação Ambiental é possível implementar os conceitos de modelagem e análise de sustentabilidade das edificações por meio do BIM 6D.

Nas disciplinas correspondentes a área de estruturas, a implementação do BIM junto ao processo de aprendizado da disciplina pode ser empregada a partir do $5^{\circ}$ semestre através de Resistência dos Materiais I, utilizando algum software para fins demonstrativos de como determinados exemplos da matéria se aplicariam a uma estrutura real. Já nas disciplinas de Estruturas de Concreto e Estruturas de Aço, sua abordagem seria mais direta, usando softwares compatíveis com a metodologia BIM e aplicando conceitos como armazenamento na nuvem, modelagem 3D e análise numérica, tais disciplinas também são essenciais para trabalhar a coordenação e a comunicação do grupo visto que os projetos não são individuais. Cabe ressaltar que atualmente é empregado pelo docente, o software Autodesk Robot na 
disciplina de Estruturas de Concreto, um software BIM utilizado para fazer análises estruturais.

No setor de transportes a maior aplicação se dá na disciplina de Projeto e Construção da Infraestrutura Viária, por razões semelhantes a área de recursos hídricos, visto que se trata de obras de infraestrutura de grande porte. Desse modo o BIM facilitaria o trabalho em grupo e aumentaria a compreensão dos alunos acerca do tema estudado ao mesmo que produziria um modelo mais preciso. Já na disciplina de Análise e Planejamento de Sistemas de Transporte, o BIM poderia ser explicado apenas de forma teórica.

$\mathrm{Na}$ área da geotecnia o BIM poderia ser empregado principalmente em Fundações, onde a modelagem 3D poderia facilitar a compreensão dos alunos e os cálculos através das análises numéricas. A disciplina de Mecânica dos Solos II poderia abordar apenas de forma introdutória o conceito, de modo a preparar os alunos para a disciplina de fundações.

A área de construção civil, foi a que teve maior aplicabilidade do BIM, que varia desde o nível introdutório/teórico até a possibilidade de desenvolvimentos de projetos que contemplem as dimensões 3D, 4D, 5D e 6D:

a) Materiais de Construção I e II: boa parte de sua ementa é formada por cálculos, entretanto há a possibilidade de introduzir o BIM com o intuito de mostrar ao discente através de uma pré-modelagem, alguns dos materiais aplicados na obra e seus métodos construtivos;

b) Projeto de construção de edifícios I e II: possuem em sua ementa a elaboração de um projeto em todas as suas etapas e, com isso, inclui a possibilidade de implantação do BIM em todas elas. A partir de 2019.1 alguns alunos da disciplina implementaram o Autodesk Revit em seus projetos por iniciativa própria, o que acabou por gerar curiosidade nos demais e a adoção em maior escala nos semestres seguintes, dadas as vantagens de concepção e agilidade em gerar pranchas e cortes;

c) Instalações elétricas: possui diversos caminhos para abordagem e diferentes softwares a serem utilizados pelo professor, entretanto por se tratar de um projeto bastante complexo para se visualizar apenas em $2 \mathrm{D}$, a modelagem $3 \mathrm{D}$ viria a fornecer um modelo mais completo e de maior compreensão tanto para execução quanto para o processo de aprendizagem;

d) Instalações Hidrossanitárias: por motivos semelhantes à disciplina de instalações elétricas, as instalações hidrossanitárias são mais passíveis de erros graves, visto que a dimensão das instalações é maior e quando há incompatibilidades com projetos estruturais por exemplo, os danos podem ser graves. A modelagem 3D quando feita, facilita a identificação de interferências, diminuindo a ocorrência de erros que só iriam ser resolvidos no canteiro de obras. Nos semestres de 2019.1 e 2019.2 foi empregado por uma docente, o software Autodesk Revit na disciplina, para modelar e dimensionar as instalações de um edifício com arquitetura também em Revit, possibilitando aos alunos um contato inicial com o BIM;

e) Gerenciamento da construção I: trabalha com todas as etapas de uma construção desde o projeto até a sua finalização e com isto, o uso do BIM seria bem aproveitado. Visto que, algumas ferramentas que a metodologia oferece, impacta diretamente a obra, 
começando pelo projeto até seu controle de material e consequentemente o seu prazo de entrega.

Nota-se que nos últimos semestres, as disciplinas de Trabalho de Conclusão de Curso I e II também podem ter relação com o paradigma BIM, visto que, dependendo da metodologia do trabalho apresentada, o BIM poderá ser aplicado como ferramenta neste estudo, por sua capacidade de abranger diferentes áreas da Engenharia Civil.

Entre as dificuldades mencionadas pelos docentes do curso para implementar o ensino do BIM em suas disciplinas, as principais são:

$\checkmark$ Falta de conhecimento aprofundado acerca do tema;

$\checkmark$ Falta de tempo para preparação de aulas que abordem o BIM;

$\checkmark$ A carga horária não comporta abordar este assunto;

$\checkmark$ A ementa da disciplina não é adequada, em termos de conteúdos e metodologia.

Cabe ainda ressaltar que no ano de 2019 foi criada a Liga Acadêmica de BIM da UFCA, partindo de iniciativa dos discentes e com auxílio de duas docentes, tem o propósito de disseminar o paradigma na universidade através de ações de ensino, pesquisa e extensão. Atualmente a liga já auxilia alunos e professores que utilizam o BIM em suas disciplinas.

\section{CONCLUSÕES}

Com esta pesquisa foi possível reconhecer e mapear as disciplinas do curso de Engenharia Civil da UFCA que possuem interface com o paradigma e apontar os possíveis pontos de atuação. Porém, a análise com todas as disciplinas do curso não exclui a possibilidade de criação de componente específicos (inclusive optativos) para debater e aplicar o BIM, mas parte da premissa de que a discussão do paradigma em diferentes momentos da formação do aluno torna-se mais abrangente e efetiva, e permite que sejam trabalhados diferentes aspectos do BIM, com diferentes professores/especialistas, possibilitando ao estudante uma formação mais integrada e completa.

A avaliação da matriz curricular já existente facilita a inserção do tema na formação do aluno, pois não demanda uma mudança radical da estrutura vigente, tal como a criação de um novo projeto pedagógico para o curso, porém deve se estabelecer um diálogo com os docentes, acerca de como o paradigma melhor se adequaria ao conteúdo ministrado na disciplina, podendo haver inclusive "disciplinas piloto" com a função de averiguar como se deu a implantação de determinado conceito específico do BIM. Este pode ser um dos primeiros passos para introduzir o BIM no curso. Deve-se considerar, entretanto, que outras questões deverão ser mudadas, em relação aos processos de ensino-aprendizagem usualmente adotados nas graduações, como o desenvolvimento da autonomia do estudante, liderar, sua proatividade, sua capacidade para trabalhar em equipes e com projetos que envolvam diferentes disciplinas.

Com relação a matriz do curso da UFCA, constatou-se que o método adotado permitiu que fosse ressaltada a interface que o curso tem com o tema, identificando diversas disciplinas que podem trabalhar conteúdos relativos ao BIM. Identificou-se que já nos primeiros semestres, ainda nas disciplinas básicas, a metodologia pode ser introduzida, sendo que questões relativas à etapa de projeto da edificação podem ser aprofundadas em semestres posteriores. Outrossim, a inserção da metodologia BIM no curso como um todo depende do 
interesse e da mobilização de um grupo multidisciplinar de professores juntamente com o apoio de indivíduos com conhecimentos abrangentes e sólidos acerca do BIM para assessorar a equipe, visando identificar as possibilidades e formas de inserção do tema no processo de aprendizado do aluno.

Identificar como será abordado a temática BIM em sua grade curricular e o tempo a ser demandado faz-se extremamente importante para uma abordagem inicial em diferentes etapas do curso, com o intuito que o aluno possa desenvolver e aprender de forma lúcida todo o conceito BIM e as suas demais ferramentas ofertadas em seus distintos níveis. Além disso, com o conceito BIM desenvolvido e bem maturado ao aluno, há a possibilidade de em parceria com algumas empresas da construção civil da região com interesse no tema, inserir o estudante no mercado de trabalho e proporcionar uma experiência profissional para o aluno e desenvolver a prática nas empresas.

Percebe-se que ainda existe um longo caminho para a efetiva adoção e consolidação do BIM nos cursos relacionados ao setor da Construção Civil. No entanto, o primeiro passo a ser consolidado é a mudança de mentalidade, visto que não se trata de "um novo Cad" e sim de toda uma cadeia de processos que vai desde a concepção do projeto até sua eventual demolição. Vale também ressaltar que o ensino do BIM não deve se sobrepor aos conceitos teóricos da disciplina, devendo se adaptar de modo a não prejudicar o desenvolvimento intelectual do profissional. Acredita-se que é possível que cada curso dê os primeiros passos para implementar o BIM, em um processo que certamente deverá ocorrer de forma incremental e em diferentes etapas.

\section{REFERÊNCIAS}

BARISON, M. B. Introdução de Modelagem da Informação da Construção (BIM) no currículo: uma contribuição para a formação do projetista. 2015. 387f. Tese (Doutorado em Engenharia de Construção Civil) - Universidade de São Paulo, São Paulo, 2015

BRASIL. Decreto $\mathbf{n}^{\mathbf{0}} \mathbf{1 0 3 0 6}$, de 2 de abril de 2020. Dispõe sobre a Estratégia Nacional de Disseminação do Building Information Modelling e institui o Comitê Gestor da Estratégia do Building Information Modelling, 2020.

CHECCUCCI, É. S.; AMORIM, A. L. Método para análise de componentes curriculares: identificando interfaces entre um curso de graduação e BIM. Pesquisa em Arquitetura e Construção, Campinas, v. 5, n. 1, p. 6-17, jan./jun. 2014. Disponível em:

https://periodicos.sbu.unicamp.br/ojs/index.php/parc/article/view/8634540/2461. Acesso em: 30 mar. 2020.

EASTMAN, C.; TEICHOLZ, P.; SACKS, R.; LISTON, K. Manual de BIM: Um guia de modelagem da informação da construção. Tradução: Cervantes Gonçalves Ayres Filho et al. Porto Alegre: Bookman, p. 84-86, 2014.

GIL, Antônio Carlos. Como elaborar projetos de pesquisa. São Paulo, Atlas S.A. 2010.

LINO, J. C.; AZENHA, M.; LOURENÇO, P. Integração da Metodologia BIM na Engenharia de Estruturas. In: ENCONTRO NACIONAL BETÃO ESTRUTURAL, Porto, 2012. Anais... Porto: FEUP, 2012.

RUSCHEL, R.; ANDRADE, M.; MORAIS, M. O Ensino de BIM no Brasil: onde estamos? Ambiente Construído. Porto Alegre, v. 13, n. 2, p. 151-165, abr./jun. 2013 